\title{
EL OTRO COMO ESTRUCTURA DISIPATIVA
}

\section{THE OTHER AS DISSIPATIVE STRUCTURE}

\author{
Alain Gabriel Jullian Montañez ${ }^{1}$ \\ al304455@yahoo.com.mx
}

Fecha de recepción: 23 agosto 2015 - Fecha de aceptación: 10 diciembre 2015

\begin{abstract}
Resumen
El otro ha sido señalado, descrito y explicado de muchas formas y desde diversas perspectivas. El esfuerzo por resolver el problema del otro ha sido tan amplio que incluso se han estado presentando explicaciones de segundo orden. Es decir, interpretaciones, acotaciones y ampliaciones de las obras de algunos de los autores más citados y reconocidos. A pesar de esto, la base material intrínseca de dicho fenómeno aún no ha sido explorada en ningún trabajo de investigación (salvo en el intento de Merleau-Ponty de percibir al otro como cuerpo, en cuyo caso fue más bien superficial el abordaje). En este artículo, se propone partir de los sistemas físicos alejados del equilibrio, denominados genéricamente como "estructuras disipativas", para entender lo que el otro es. Una vez que el otro ha sido considerado y explicado en estos términos, algunas implicaciones del fenómeno de la alteridad se dejan ver. La principal implicación tiene que ver con el cómo el otro influye en nosotros, dejando en claro que el otro no solamente nos permite autodefinirnos. Más bien, participa en nuestra propia construcción, desarrollo y evolución. Así, el valor que se le asigna al otro puede haber sido minimizado a través de las explicaciones tradicionales que no consideran la base material de la alteridad.
\end{abstract}

Palabras clave: otro, alteridad, energía, termodinámica, estructura disipativa, entropía, identidad.

\begin{abstract}
The other has been pointed out, described and explained in many ways and from many perspectives. The effort to solve the problem of the other has been as wide that even second order explanations are showing up. That is to say, interpretations, comments and extensions about the work of some of the most quoted and recognized authors. Despite this, the intrinsic material foundation of this phenomenon hasn't been explained yet in any research work (except from the Merleau-Ponty's attempt to perceive the other as body, which was a very shallow approach). This article proposes starting from the physical systems far from equilibrium, generically named dissipative structures, to understand what the other is. Once the other has been considered and explained in these terms, some implications of the otherness phenomenon are outlined. The main implication has to do with "How does the other affect us?" establishing that the other doesn't only allow us to be self-defined. It is rather involved in our own construction, development and evolution. Thus, the value ascribed to the other might have been minimized through traditional explanations, which don't consider the material basis of otherness.
\end{abstract}

Key words: other, otherness, energy, thermodynamics, dissipative structure, entropy, identity.

1 Centro de Investigaciones Interdisciplinarias Sobre Desarrollo Regional de la Universidad Autónoma de Tlaxcala. 


\section{Resumé}

L'autre a été signalé, décrit et expliqué de plusieurs façons et dès diverses perspectives. L'effort pour resoudre le problème de l'autre a été si approfondi que même explication de deuxième ordre commencent à se présenter. C'est à dire, interprétations, annotations et approfondissements des ouvres de quelques auteurs des plus cités et reconnus. Malgré ça, la base matérielle intrinsèque de tel phénomène n'a pas été exploré encore dans aucun travail de recherche (sauf l'essai de Merleau-Ponti pour percevoir l'autre comme corps, dont le cas a été très superficiel son abordage). Dans cet article, se propose partir des systèmes physiques hors de l'équilibre, dénommés génériquement "structures dissipatives", pour comprendre ce que l'autre est. Une fois que l'autre a été considéré et expliqué en ces termes, quelques implications du phénomène d'altérité se laissent voir. L'implication principale a à voir avec la forme comme l'autre influence sur nous, en faisant comprendre que l'autre ne seulement nous permet de nous définir. Plutôt, il participe dans notre même construction, développement et évolution. Ainsi, le valeur attribué à l'autre peut avoir été minimisé à travers des explications traditionnelles qui ne considèrent pas la base matérielle de l'altérité.

Mots clé: autre, altérité, énergie, termodinámica, structure dissipative, entropie, identité.

\section{Introducción}

Que el "Otro" no sea nadie propiamente hablando, ni usted ni yo, significa que es una estructura que se encuentra solamente efectuada por medio de términos variables en los diferentes mundos perceptivos - yo para usted en el suyo, usted para mí en el mío-. No basta siquiera con ver en otro una estructura particular o específica del mundo perceptivo en general; de hecho, es una estructura que funda y asegura todo el funcionamiento del mundo en su conjunto. Y es que las nociones necesarias para la descripción del mundo (...) permanecerán vacías e inaplicables, si el "Otro" no estuviera ahí, expresando mundos posibles (Gilles Deleuze).

El otro ha sido señalado, descrito y explicado de muchas formas y desde diversas perspectivas. Así, autores como Emmanuel Levinas, Carlos Skliar o Tzvetan Todorov, entre muchos otros, intentaron dar cuenta del fenómeno de la alteridad (Levinas, 1988; 1993; 2002; Skliar, 2002; Todorov; 2001).

El esfuerzo por resolver el problema del otro ha sido tan amplio que incluso empiezan a presentarse explicaciones de segundo orden. Es decir, interpretaciones, acotaciones y ampliaciones de las obras de algunos de aquellos autores que son más reconocidos o destacados. Por ejemplo, Koolhaas Jurgens en 1972 publicó la explicación que él hace sobre la obra de Lacan en ¿Quién es el otro? Del mismo modo, Navarro en 2008, presentó El «rostro» del otro: Una lectura de la ética de la alteridad de Emmanuel Lévinas, en donde intenta redefinir la obra de aquel. $\mathrm{O}$, más recientemente, Pirni hizo púbico un artículo, en 2013, titulado: “¿Contra Schmitt? Modelos de alteridad para la convivencia”, en donde reelabora las ideas de Schmitt para proponer un marco de convivencia amplio y pertinente en un mundo como el actual (Pirni, 2013).

A pesar del esfuerzo realizado para entender al otro, la base material intrínseca de dicho fenómeno aún no ha sido explorada. La única excepción a esto es la idea de Maurice Merleau-Ponty de partir del cuerpo para definir las relaciones con el otro (Merleau-Ponty, 1994). Sin embargo, esta propuesta termina por alejarse del fundamento material del que, de manera muy escueta, parte y acaba por enfocarse en el cómo los sentidos ayudan a percibir y relacionarse con el otro. De hecho, resulta significativo que en algunas ocasiones él mismo cuestiona la pertinencia del abordaje físico. Por supuesto, la física de tiempos de este autor no tenía los elementos para dar cuenta de muchos de los fenómenos hipercomplejos existentes ya que era, todavía, una física de sistemas ideales.

Salvo esa línea de pensamiento, que inicia en el cuerpo, la discusión del otro suele centrarse en los intangibles; es decir, en el cómo se designa o identifica al otro, o al cómo se debe tratar al otro. Esto es, asumiendo los ejes epistémico y axiológico, para hablar en términos de Todorov (2001). En todo caso, no es del interés de este artículo la interacción misma con el otro desde el punto de vista de los sentidos. Ya que la discusión no debe (ría) girar en torno a si la relación es con intermediarios o sin 
intermediarios, si es mediata o inmediata, a través de los sentidos o sin ellos, el quid del asunto es que el otro existe y el cómo es que existe y cómo nos afecta su existencia, sea esta percibida, por nosotros, o no.

\section{Diversas perspectivas, conclusiones semejantes}

Aquí, conviene voltear la mirada a las explicaciones existentes sobre el otro. En este sentido, el artículo de revisión que publicó González-Silva en esta revista resulta indispensable (González-Silva, 2009). En dicha obra, se hace una exploración exhaustiva sobre los trabajos sobre la alteridad desde 1970 hasta la fecha de la publicación del trabajo. Además, se bosqueja lo que, para el autor, es el fundamento de dicho tema desde las ideas de Platón hasta las de Hegel.

De los casi 50 trabajos que incluye la revisión, se destacan los esfuerzos para definir la relación con el otro y el sentido pragmático de algunas de las investigaciones. Lo anterior, particularmente, desde 2004; y no debido a que antes de ese año no se haya buscado alguna utilidad al tema de la alteridad, sino a que se estableció cierta tendencia hacia la praxis. Por ejemplo, González (2007 y 2008), lo utilizó como herramienta para abordar problemas vinculados con la familia, mientras que Pérez (2006) centró su atención en su aplicación a la pedagogía. Sin embargo, el otro se sigue presentando, a grandes rasgos, como inmóvil, como algo estático.

¿Qué sucede con las perspectivas de alteridad fuera de la revisión de González- Silva? Se desenvuelven más o menos en el mismo sentido. Verano-Gamboa (2012), Pirni (2013), Monteagudo (2013), por citar sólo tres trabajos recientes, continúan mostrando al otro de forma más bien estática. Un caso diferente, y por lo mismo notable, es la obra de Carlos Skliar (2002). Para él, el otro no es, el otro está siendo. De hecho, ve necesario replantear tiempo y espacio para hacer su propuesta pedagógica. El único inconveniente de la obra de Skliar es que no abunda sobre el particular.

Resumiendo. Los trabajos referidos sobre alteridad tienen la virtud de definir la relación con el otro, e incluso aplicar a problemas concretos el conocimiento existente sobre el tema. No obstante, tienen el inconveniente que, de facto, presentan al otro de manera estática; siendo esta su principal limitación. Se plantea, entonces, la pregunta ¿cómo explicar al otro de forma tal que se supere esta debilidad?

\section{Percibiendo al otro de forma dinámica}

Para responder la pregunta anterior, en este artículo, se propone partir de un enfoque diferente vinculado con las ideas del físico y premio Nobel Ilya Prigogine para entender lo que el otro está siendo. Por tanto, el fundamento de la propuesta presentada aquí son los sistemas físicos alejados del equilibrio termodinámico, denominados genéricamente como "estructuras disipativas".

Antes de continuar, resulta pertinente hacer una acotación. El que se utilicen modelos de la física del no equilibrio no es un intento de exportar sin miramientos principios de otras disciplinas a las ciencias sociales. En este sentido, se tiene muy claro que no se puede reducir la totalidad de los procesos sociales a explicaciones de otras áreas del saber. Así, se manifiesta una total concordancia con autores como Georgescu-Rogen o el mismo Ilya Prigogine (Prigogine, 2001; Georgescu-Rogen, 1981). Lo único que se plantea aquí es que la termodinámica impone límites materiales a las formas de vida y dichos límites repercuten en nuestras relaciones con el otro y, por eso, es necesario explorarlos. Este planteamiento sigue lo propuesto por Adams (2007) en el sentido que la segunda ley de la termodinámica es un instrumento de trabajo directamente aplicable a los fenómenos sociales sin que medie para esto alguna metáfora. En el fondo, esto es una derivación de las ideas del antropólogo Leslie White. Él propuso, básicamente, que todo en el universo puede ser explicado en términos de la energía (White, 1943). Esto, se asume, incluye al fenómeno de la alteridad. Por otro lado, el funcionamiento y vida de las estructuras disipativas se sustenta en los flujos de energía lo cual está en estrecha relación con el planteamiento de White. 
Las estructuras disipativas son sistemas autoorganizados que dependen de los flujos de materia y energía para su permanencia. Ante estos flujos, las estructuras disipativas pueden permanecer gracias a que poseen la propiedad de homeostasis y la habilidad para saltar de un estadio a otro en una sucesión que les permite continuar disipando energía, aunque con modificaciones entre los estadios. Esto, quiere decir que estas estructuras contienen en sí mismas los elementos para mantenerse durante cierto periodo de tiempo, siempre y cuando continúen disipando energía (Blackburn, 1973; Prigogine, 1977; Tyrtania, 1999). La existencia de estas estructuras fue propuesta por Ilya Prigogine en la década de 1960 (Zhu, 2012).

Como puede verse, las estructuras disipativas son un fenómeno complejo, es por eso que en el universo hay una gran diversidad de ellas. Algunos ejemplos de estructuras disipativas que nos pueden resultar familiares son la flama de una vela, los seres vivos, los ecosistemas y las ciudades (Schneider et al, 1994; Adams, 2001; Margulis, 2000). Esta variedad que presentan las estructuras disipativas ha obligado a los que las estudian a hacer clasificaciones de ellas. Richard N. Adams (2001) las organiza, a grandes rasgos, en tres grupos; químicas, biológicas y sociales. Para fines de este trabajo se destacan las biológicas. Esto es, el otro se considera una estructura disipativa de tipo biológico.

\section{El otro como estructura disipativa}

Una vez que el otro ha sido considerado y presentado en términos de las estructuras disipativas, algunas implicaciones del fenómeno de la alteridad pueden percibirse con mayor facilidad. Las más conspicuas son: a) la existencia del otro es independiente de la designación que se pueda hacer de él, b) el otro es un generador de entropía, c) el otro posee la capacidad para definirnos, d) el otro posee la capacidad para construirnos, e) el otro no debe ser concebido simplemente como lo que está afuera. A continuación se abunda en cada uno de estos puntos.

La existencia del otro es independiente de la designación que se pueda hacer de él. Esto se puede aseverar debido a que la existencia del otro está en función de los intercambios de materia y energía que realice para poderse mantener alejado del equilibrio termodinámico (es decir, la muerte). Enfocando el punto desde las ideas de Levinas: La invisibilidad no indica una ausencia de relación; implica relaciones con lo que no está dado, de lo cual no hay idea. (Levinas, 2002). Así, la capacidad para percibir al otro depende de dos cosas, de nuestros sentidos (aunque sean amplificados con medios tecnológicos) y de que la señal enviada por el otro sea proclive a ser percibida por nosotros. Si alguna de estas dos falla, no hay posibilidad de percibirlo. Pero de ninguna manera significa que el otro no exista, ya que, como se indicó, su existencia se debe única y exclusivamente a su capacidad de intercambio de materia y energía.

El otro como generador de entropía. La estructura disipativa se autoorganiza y genera entropía en el medio, esto es una condición sine qua non de ella. Hay que destacar, sin embargo, que esta disipación de calor que sucede al transformar energía no solo genera caos o desorden (Tyrtania, 2009; Prigogine, 2001). Es por esto que Prigogine destaca repetidas veces en su obra la capacidad creadora de dicha entropía (Spire, 2000). Es decir, el que el otro sea un generador de entropía lo convierte ipso facto en un ente dual, ambivalente, que modificará su medio (nuestro entorno compartido) a veces para perjuicio de las demás estructuras disipativas o a veces para beneficiarlas. De paso, subraya su dinamismo; ya que el que el otro sea un generador de entropía obliga a asumirlo como en constante construcción. Esto significa que se le tiene que entender en cambio adaptativo, siendo, llegando a ser, pero nunca se le puede aceptar como algo fijo o estático.

La capacidad del otro para definirnos. Esta es una de las virtudes del otro que ha sido descrita y comentada por varios autores; uno de ellos es Pirni (2013). En su trabajo se destacan dos formas en que el otro aporta a nuestro autoconcepto y a la definición de nuestras propias características. Él lo maneja como dos formas de alteridad, el muro y el espejo. 
¿Cómo nos define el otro? Al constituirse un límite externo para nosotros (Pirni lo llama figura de alteridad-muro), nos permite definirnos en relación con nuestro alcance. Además, el otro nos ayuda a identificarnos porque se puede ver en él características de nosotros mismos (deseables o no). A esto, Pirni lo denomina como figura de alteridad-espejo (2013). Estas son las dos maneras en que el otro nos define que están de acuerdo con la tesis que se plantea en este texto, ya que las estructuras disipativas poseen fronteras bien definidas (que concede la posibilidad del "muro") y "la vida implica sensibilidad y capacidad de elección, incluso en sus niveles más primarios" (Tyrtania, 2009:44). Lo cual sugiere la posibilidad del espejo.

La capacidad del otro para construirnos. El otro nos construye no únicamente en el sentido en que se enfocó el punto anterior; es decir, como coadyuvante para la propia identificación. No, su capacidad de construirnos va más allá; literal y biológicamente nos construye.

Esta construcción que el otro hace de nosotros, que se puede denominar biológica, parte de las interacciones necesarias con el medio. Se dijo que al ser estructura disipativa, necesariamente modifica el ambiente en el que se desenvuelve, en el que nos desenvolvemos (el otro y nosotros). Así, por cuanto nos condiciona debido su capacidad de limitar, por competencia, nuestra posibilidad de hacernos de recursos (sean materiales, energéticos o de información), el otro nos construye. Asimismo, existen recursos que nos hará accesibles debido a su actividad, y nosotros a los otros debido a nuestras acciones, a nuestros propios intercambios energéticos y materiales. Entonces, en realidad, no solamente nos limitamos mutuamente, sino que nos creamos recíprocamente. Por tanto, el otro no es solo lo externo, es la causa que provoca que mi medio cambie, que me obliga a adaptarme a un medio en constante cambio por la acción intrínseca de la existencia del otro. La anterior sería una postura semejante a la interanimalidad que propone Ramírez-Barreto (2010) pero partiendo de la física de Prigogine.

En términos de las características propias de las estructuras disipativas, el otro desarrolla nuestra capacidad de homeostasis. ¿Cómo es esto? La homeostasis puede ser definida de forma sencilla como el conjunto de fenómenos de autorregulación, que conducen al mantenimiento de la constancia en la composición y propiedades del medio interno de un organismo. Entonces, si esos flujos que nos resultan necesarios son restringidos (coartados) por el otro, en realidad lo que se está provocando es poner a prueba nuestra homeostasis, ya que, de manera práctica, el otro funciona como un regulador (cualitativo y cuantitativo) de los flujos que nos son accesibles; y es, justamente a esos flujos, a los que reacciona nuestro organismo intentando mantener su composición y propiedades.

El otro no es simplemente lo que está afuera. Ver al otro como estructura disipativa biológica implica asumirlo como el que está afuera, no como lo que está afuera. Esto es pertinente porque lo que está afuera es inerte, el que está afuera puede actuar. Esta es una de sus características principales, su capacidad de acción; es justamente aquella que utiliza para construirnos. Eso es, en realidad, lo relevante, porque se le des-cosifica por medio de reconocer su capacidad para la acción.

El destacar la capacidad de actuar no es un asunto menor, ya que repercute en el núcleo de la explicación acerca del otro. ¿Por qué? Porque cuestiona las explicaciones pro-cartesianas del otro. Es decir, el otro no es otro porque sea un cogito, no es otro porque piensa (porque de hecho muchas de sus acciones no son reflexionadas, a veces, incluso, son automáticas), es otro porque actúa. En la medida en que actúa, modifica nuestro entorno y a nosotros mismos y a él mismo. El solo pensar, meditar o racionalizar algo no nos hace otro porque esas son acciones propias del mismo son introspectivas por cuanto suceden y se mueven en el interior. Es decir, por mucha conciencia que se tenga de uno mismo eso, por sí solo, no nos hace otro porque la autoconciencia es conciencia de mí, de lo que está adentro, entonces eso, a lo sumo me hace mismo, pero no otro. En cambio, la acción sucede hacia afuera; siempre con relación a la alteridad, a las alteridades. Incluso la acción fisiológica que se asume privada, íntima, propia, en realidad, como se expuso anteriormente, se vincula al exterior, es mediada desde el exterior. 


\section{Conclusiones}

El considerar al otro desde su fundamento físico-material, es decir, como estructura disipativa, permite distinguirlo con mayor claridad, ya que, al percibirlo desde una perspectiva no tan limitada, se hacen evidentes algunas virtudes que posee. Bajo esta forma de entender al otro, se le presenta como independiente, creador, como ayudante nuestro, pero, sobre todo, como capaz de modificar el entorno y actuar.

Así, el valor que se le asignaba al otro puede haber sido minimizado a través de las explicaciones tradicionales que no consideran la base física de la alteridad y que lo presentan como estático. Quizá, al volcar todo el esfuerzo por conceptualizarlo, por definirlo, por identificarlo se dejó un poco de lado su capacidad para la acción; es decir, el acento se puso en describirlo, no en el dinamismo que implica el hecho de estar siendo otro.

\section{Agradecimientos}

Deseo agradecer al Consejo Nacional de Ciencia y Tecnología (Conacyt) por financiar esta investigación a través de las Estancias Posdoctorales Nacionales 2013(3), así como al Centro de Investigaciones Interdisciplinarias para el Desarrollo Regional (CIISDER) de la Universidad Autónoma de Tlaxcala por permitirme desarrollar este trabajo en sus instalaciones. Agradezco, también, a la maestra en Ciencias Claudia Patricia González Lozano por la traducción del resumen a los idiomas inglés y francés. Por último, incluyo en estos agradecimientos a los dictaminadores anónimos de esta revista que enriquecieron notablemente el artículo con sus comentarios y sugerencias.

\section{Referencias}

Adams Richard Newbold (2001). El octavo día. La evolución social como autoorganización de la energía. Editado por la Universidad Autónoma Metropolitana. México, D.F. 411 p.

Adams, Richard Newbold. (2007). La red de la expansión humana. CIESAS. México, D.F. 270p.

Blackburn Thomas R. (1973). Información y ecología de la academia. p 21-42. En L. Tyrtania, Termodinámica de la supervivencia para las ciencias sociales. Universidad Autónoma Metropolitana. México D.F.

Georgescu-Roegen, Nicholas. (1981). Afterword. p. 119-130. En L. Tyrtania. Termodinámica de la supervivencia para las ciencias sociales. Universidad Autónoma Metropolitana. México D.F.

González-Silva, Freddy. (2009). Alteridad y su itinerario desde las perspectivas multidisciplinares. Revista Reflexiones. 88(1): 119-135.

Koolhaas-Jurgens Gilberto. (1972). ¿Quién es el otro? Revista uruguaya de psicoanálisis. 13 (4) Recurso en línea, consultado el día 8 de Julio de 2014: http://www.apuruguay.org/apurevista/1970/1688724719711972130401.pdf

Levinas Emmanuel (1988). La Huella del otro. Taurus, México D.F. 116 p.

Levinas, Emmanuel (1993). El tiempo y el otro. Barcelona, Paidós. 144 p.

Levinas, Emmanuel (2002). Totalidad e infinito. Ensayo sobre la exterioridad. Sígueme. Salamanca. $315 \mathrm{p}$.

Margulis Lynn, Sagan Dorion (2000). What is life? Simon \& Shuster. Canada 297 p.

Merleau-Ponty, Maurice (1994). Fenomenología de la percepción. Planeta-Agostini. España. 470 p.

Monteagudo Cecilia (2013). El diálogo posible entre dos tradiciones. Entre la“escucha del otro" y el "principio de caridad". Areté. 25(2): 267-282.

Navarro Olivia (2008). El «rostro» del otro: Una lectura de la ética de la alteridad de Emmanuel Lévinas. Contrastes. Revista Internacional de Filosofía, 13: 177-194. 
Prigogine Ilya. (1977). Tendencias a largo plazo y evolución de la complejidad. p. 43-118. En L. Tyrtania, Termodinámica de la supervivencia para las ciencias sociales. Universidad Autónoma Metropolitana. México D.F.

Prigogine Ilya (2001). Tan solo una ilusión. Tusquets editores. Barcelona. 325 p.

Prigogine, Ilya (2001). El fin de las certidumbres. Editorial Taurus. España. 230 p.

Pirni, Alberto. (2013). ¿Contra Schmitt? Modelos de alteridad para la convivencia. Bajo Palabra, Revista de filosofía. II época, 8: 335-352.

Ramírez-Barreto, Ana Cristina. (2010). Ontología y antropología de la interanimalidad. Merleau-Ponty desde la perspectiva de Tim Ingold. AIBR. Revista de Antropología Iberoamericana. 5(1): 32-57.

Schneider, E.D. Kay, J.J. (1994). "Life as a manifestation of the second law of Thermodynamics". Mathematical and computer Modelling. 19 (6-8): 25-48.

Skliar Carlos (2002). ¿Y si el otro no estuviera ahí? Notas para una pedagogía (improbable) de la diferencia. Miño y Dávila. Buenos Aires 160 p.

Spire Arnaud. (2000). El pensamiento de Prigogine. Andrés Bello. Santiago. 191 p.

Todorov Tzvetan (2001). La conquista de América. El problema del otro. Siglo XXI. México, D.F. 277 p.

Tyrtania Leonardo (1999). Termodinámica de la supervivencia para las ciencias sociales. Universidad Autónoma Metropolitana. México D.F. 230 p.

Tyrtania Leonardo (2009). Evolución y sociedad. Termodinámica de la supervivencia para una sociedad a escala humana. Universidad Autónoma Metropolitana. México D.F. 368 p.

Verano-Gamboa Leonardo (2012). El lugar del otro: El problema de la alteridad en la filosofía de Merleau-Ponty. Universitas Philosophica. 58, año 29: 251-275.

White Leslie. (1943). Energy and the evolution of culture. American Anthropologist new series. 45(3): 335-356.

Zhu,Q., Liu,X., Zhang H. (2012). Research on the safety economic management system based on the dissipative structure theory. Procedia Engineering 45: 259-263. 
